\title{
ANTIBIOTIC USE IN PEDIATRIC INFECTIONS; A STUDY IN TERTIARY CARE HOSPITAL \\ Praveena Gungam ${ }^{1}$, Y. Sunil Kumar Yadav ${ }^{1}$, Sunil Junapudi ${ }^{2}$ \\ ${ }^{1}$ Department of Pharmacology, Osmania Medical College, Koti, Hyderabad, Telangana, India- 500095. \\ ${ }^{2}$ Dept. of. Pharmaceutical Chemistry, Geethanjali College of Pharmacy, \\ Cherryal, Keesara, Medchal, Telangana, India-501301.
}

\section{ABSTRACT}

Objectives: Antibiotics are frequently used in tertiary care hospitals. We conducted an observational study on children admitted to a teaching hospital in south India, to make a profile of antibiotics use and suspected adverse drug reactions (ADRs) owing to them. Methods: Hospitalized children of either sex, aged between 1 month and 12 years, were inspected. Baseline demographic and clinical features, duration of hospital stay, antibiotics received in hospital along with dosing and indications and interest of suspected ADRs attributable to their use were recorded. Every patient was followed up till discharge, admission to the Pediatric Intensive Care Unit, or passing. Results: Over the year and a half report period 364 confirmations were screened. The prevalence of Antibiotics use was $80.22 \%$. The majority of the 292 children who received Antibiotics were males (63.35\%). Median age was 35 months, five children died. In most instances, either two (41\%) or a single antibiotic (37.32\%) was used. Ceftriaxone, co-amoxiclav, amikacin, vancomycin, and ampicillin were predominantly used. Antimalarials, antivirals and antiprotozoals were used occasionally. Average number of Antibiotics per patient was $2.2 \pm 1.1$ the majority (81.15\%) were by parenteral route and initial choice was usually empirical. Prescriptions were usually in generic name. The antibiotic treatment went somewhere in the range of 1 and 32days, with a middle of 8 days. Five ADRs were noted of which half were skin rash and the rest loose stools. Conclusions: The profile of Antibiotic utilize is comprehensively like prior Indian investigations. Apparent overuse of multiple Antibiotics per prescription and the parenteral route requires exploration. Antibiotics are being used empirically in the absence of policy. ADRs to Antibiotics are occasional and usually mild. The benchmark information can serve in situation analysis for antibiotic prescribing guidelines.

KEYWORDS: Antibiotic; Pediatric infections; Adverse drug reactions; Tertiary care hospital.

\section{INTRODUCTION}

This is evidenced by the WHO report where it was exhibited that in creating nations, half of all popular upper respiratory tract diseases and viral infection cases got anti-infection agents improperly while just $70 \%$ of all pneumonia cases, which warrant anti-infection treatment, antibiotics[1]. This inappropriate antibiotic utilize has numerous outcomes including serious morbidity and mortality coming about because of wrong dose, antagonistic medication response, and expanded Antibiotic obstruction coming about because of abuse of anti-infection agents. In 2011, the WHO expressed "if no action today, no cure tomorrow" [2] in an effort to emphasize to the consequence of the widespread and inadvertent use of antibiotics and the need for immediate action to preserve antibiotics for future use.

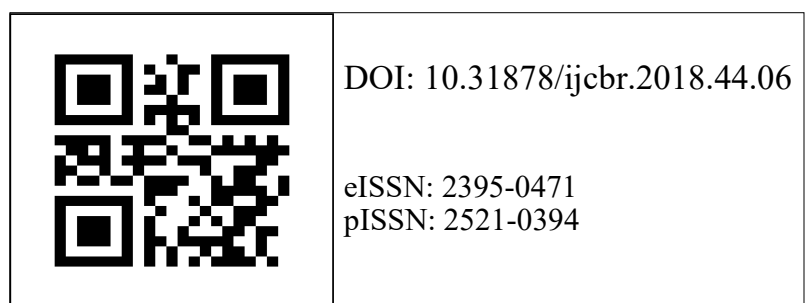

Infectious diseases represent a major cause of morbidity and mortality in India and are responsible for a large proportion of hospital admissions, particularly in children. Antibiotics and other Antibiotics, therefore, constitute an important category of drugs, both in the community and in hospitals. There is considerable evidence linking indiscriminate use of Antibiotics to altered susceptibility patterns among infectious organisms and often frank resistance[3]. We face huge challenges in rational use of Antibiotics starting with general lack of awareness and unsatisfactory levels of personal hygiene and environmental sanitation to lack of surveillance mechanisms for monitoring Antibiotic use and resistance, mostly empirical use of antibiotics due to dearth of microbiology laboratory support, absence of or ineffective antibiotic use policies in most healthcare settings and nonhuman use of Antibiotics [4 $-6]$.

The nature and pattern of Antibiotic prescribing changes with time as spectrum of pathogens change and new Antibiotics are introduced. In India, Antibiotics may account for $50 \%$ of total value of drugs sold, but the prevalence of Antibiotic use has varied across surveys [7]. With widespread use of antibiotics, the prevalence

Correspondence: Dr. Y. Sunil Kumar Yadav, Department of Pharmacology, Osmania Medical College, Hyderabad, Koti, Hyderabad, Telangana, India- 500095. Email: ysunil.omc@gmail.com 
of resistance also increases. The association of resistance with the use of Antibiotics agents has been documented in both in-and-outpatient settings [8,9]. The occurrence of adverse drug reactions (ADRs) is another problem with Antibiotic use. ADRs in pediatric population may have relatively more severe effect than adults leading to significant morbidity among children [10]. A systematic review published some time back reported the incidence of pediatric ADRs at $9.5 \%$, responsible for $2.1 \%$ of hospital admissions, with $39.3 \%$ of them being life?threatening [11]. A study of ADR burden in children in South India found that ADRs occurred more among infants and antibiotics were commonly implicated [12].

Rational use of Antibiotics in the long run, therefore, demands continuous survey of Antibiotic use and related ADR monitoring [13]. Patterns of use in children need to be studied separately as they will vary from use in adults. However, there is limited data in this respect from India and no recent data from the Southern region. With this background, we planned to observe Antibiotic use in pediatric medicine ward of our tertiary care hospital to generate data regarding Antibiotic utilization and related ADRs.

\section{MATERIALS AND METHODS}

\section{Study design: Descriptive study}

Ethics approval: Institutional Ethics Committee approval was obtained beforehand. Written informed consent was obtained from a parent or legal guardian, with the provision of a witness when the guardian was illiterate.

Study duration: Study was carried out over period of 18 months (February 2016-August 2017)

Study place: The study was carried out among inpatients admitted to the pediatric medicine ward of a tertiary care teaching hospital (Osmania General Hospital, Hyderabad).

The Pediatrics Medicine Department admits 8-14 patients daily on an average.

Inclusion criteria: Subjects of both sex and age between 1 month to 12 years were recruited into if they're in house prescriptions contained Antibiotic agents.
Exclusion criteria: Seriously ill children were excluded.

Sampling method: Sampling was purposive. Recruitment was done in 1-2 days per week and every effort was made to recruit all children admitted on a study day if they satisfied inclusion criteria. The recruitment days corresponded to the fixed admission days of the pediatrician investigator, and once recruited the child was followed up till discharge, shifting to the Pediatric Intensive Care Unit (PICU), or death. Although the recruitment of new subjects was done on fixed days of the week, the researchers visited the wards as often as required to keep complete track of the medication being used by every recruited subject.

Sample size: A total of 292 patients admitted to the pediatric medicine ward were recruited during the study period.

Methodology: Antibiotics are supplied free in the hospital. Our operational definition of antibiotic agent included synthetic as well as naturally obtained drugs that attenuate microorganisms, subsequently covering all antibacterial, antifungal, antiviral, and antiprotozoals agents. Standard statistic and clinical features, duration of hospital stay, antibiotics received in the hospital along with dosing and indication and details of suspected ADRs attributable to their use were recorded. Information was captured on a structured case report form. ADR data were captured on the ADR monitoring form of Pharmacovigilance Programme of India (PvPI) [14].

Statistical analysis: The data have been summarized by routine descriptive statistics, namely mean and standard deviation for numerical variables, additionally median and interquartile range for skewed variables and counts and percentages for categorical variables. The $95 \%$ confidence interval $(\mathrm{Cl})$ has been presented for key figures. Origin Pro 7.6 statistical software was used for analysis.

\section{RESULTS}

During the 18 months study, a total of 292 patients admitted to the pediatric medicine ward were recruited. These 292 came from 364 eligible subjects; that is patients admitted to the pediatric ward on the study days if they consented to be part of the study. Of them, 72 were not given Antibiotic prescriptions. Thus, the

Table 1. Anthropometric data for the study population

\begin{tabular}{|l|l|l|l|}
\hline Anthropometric parameter & Range & Mean \pm SD & Median (IQR) \\
\hline Weight at admission $(\mathrm{kg})$ & $1.5-32$ & $13.6 \pm 5.8$ & $11.03(6.8-18.9)$ \\
\hline Height at admission $(\mathrm{cm})$ & $40.0-135.0$ & $87.3 \pm 9.8$ & $85.0(65.0-109.8)$ \\
\hline Birth weight $(\mathrm{kg})$ & $0.8-3.6$ & $2.4 \pm 1.1$ & $2.2(2.0-2.5)$ \\
\hline Head circumference $(\mathrm{cm})$ & $28.0-58.0$ & $38.6 \pm 3.76$ & $39.0(38.0-40.0)$ \\
\hline
\end{tabular}

${ }^{*}$ Measured in subjects aged 12 months or less. IQR=Interquartile range, SD=Standard deviation

Over the 18 months study, the total number of antibiotic agents prescribed to the 292 children was 589 . Table 2

provides a snapshot of this antibiotic use. 
Table 2. Antibiotic use in study subjects

\begin{tabular}{|l|l|}
\hline Parameter & \multicolumn{2}{l|}{ Value } \\
\hline Total numbers of antibiotic agents: prescribed & \multicolumn{2}{l}{589 in 292 cases } \\
\hline Number of antibiotics per patient: & $1-12$ \\
\hline Range & $2.2 \pm 1.1$ \\
\hline Mean & $2.0(1-2)$ \\
\hline Median & \multicolumn{2}{|l|}{} \\
\hline Duration of antibiotic treatment (excluding anti tubercular drugs) (days): & $1-32$ \\
\hline Range & $7.1 \pm 5.8$ \\
\hline Mean & $8.0(4-8)$ \\
\hline Median & \multicolumn{2}{|l}{} \\
\hline Duration of hospital stay (days): & $1-38$ \\
\hline Range & $8.8 \pm 6.6$ \\
\hline Mean & $8.0(5-11)$ \\
\hline Median & 82.78 \\
\hline Percentage of antibiotics as injections: & \\
\hline
\end{tabular}

$I Q R=$ Interquartile range

Table 3: System wise breakup of individual antibiotic use in 265 children

\begin{tabular}{|c|c|c|c|c|c|c|c|c|c|c|c|}
\hline & RTI & GIT & CVS & CNS & $\begin{array}{l}\mathrm{GU} \\
\mathrm{T}\end{array}$ & Sepsis & SST & $\begin{array}{l}\text { Other } \\
\text { INF }\end{array}$ & Fever NOS & TOX & $\begin{array}{l}\text { To- } \\
\text { tal }\end{array}$ \\
\hline Cephalosporins* & 62 & 19 & 23 & 36 & 38 & 6 & 3 & 4 & 18 & 31 & 240 \\
\hline $\begin{array}{l}\text { Other }-\beta \text {-lactams } \\
+ \text { vancomycins }\end{array}$ & 59 & 8 & 18 & 26 & 28 & 7 & 3 & - & 11 & 13 & 173 \\
\hline Amikacin & 23 & 5 & 13 & 8 & 7 & 3 & - & - & 6 & 3 & 68 \\
\hline Metronidazole & - & 8 & - & 4 & 5 & 2 & - & - & - & 5 & 24 \\
\hline Fluoroquinolones & 3 & 4 & 3 & 2 & 6 & - & - & - & - & - & 18 \\
\hline Antitubercular & - & - & - & 16 & - & - & - & - & - & - & 16 \\
\hline Macrolides & 6 & - & 3 & 1 & 3 & - & - & - & 1 & 3 & 17 \\
\hline Aciclovir & - & - & - & 5 & 1 & - & - & - & - & 4 & 10 \\
\hline Linezolid & 1 & - & - & 3 & - & 2 & - & - & - & 3 & 9 \\
\hline Antimalarials & - & - & - & 3 & - & - & - & 5 & 1 & - & 9 \\
\hline Co?trimoxazole & - & - & - & - & 1 & - & - & - & - & - & 1 \\
\hline Mupirocin & - & - & - & - & - & - & 2 & - & - & - & 2 \\
\hline Amphotericin B & - & - & - & 2 & - & - & - & - & - & - & 2 \\
\hline Total & 154 & 44 & 60 & 106 & 89 & 20 & 8 & 9 & 37 & 62 & 589 \\
\hline
\end{tabular}

*The cephalosporins included ceftriaxone (198), cefotaxime (10), cefpodoxime (9), cefoperazone (9), cefipime (6), cefixime (3), cefuroxime (3), cefalexin (1) and ceftazidime (1); **This group included co-amoxiclav (74), ampicillin (28), piperacillin-tazobactam (14), amoxicillin (8), meropenem (14), penicillin $V$ (3), benzathine penicillin (1), imipenem (1) and vancomycin (30); CNS =Central nervous system, CVS=Cardiovascular system, Fever NOS=Fever not otherwise specified, GIT=Gastrointestinal tract, GUT=Genitourinary tract, INF=Infection, RTI=Respiratory tract infections, SST=Skin and soft tissue infection, TOX=Toxicities (poisonings) and miscellaneous

prevalence of antibiotic use was $80.22 \%$ (95\% Cl $75.0 \%$ $-85.0 \%)$.

Among these 292 children, 185 (63.35\%) were males. The majority of the children belonged to the preschool age group, with the median age at 35.0 (interquartile range 8.0-96.0) months. The anthropometric data are summarized in Table 1 and indicates that the children were of average height and weight in consonance with their age. 


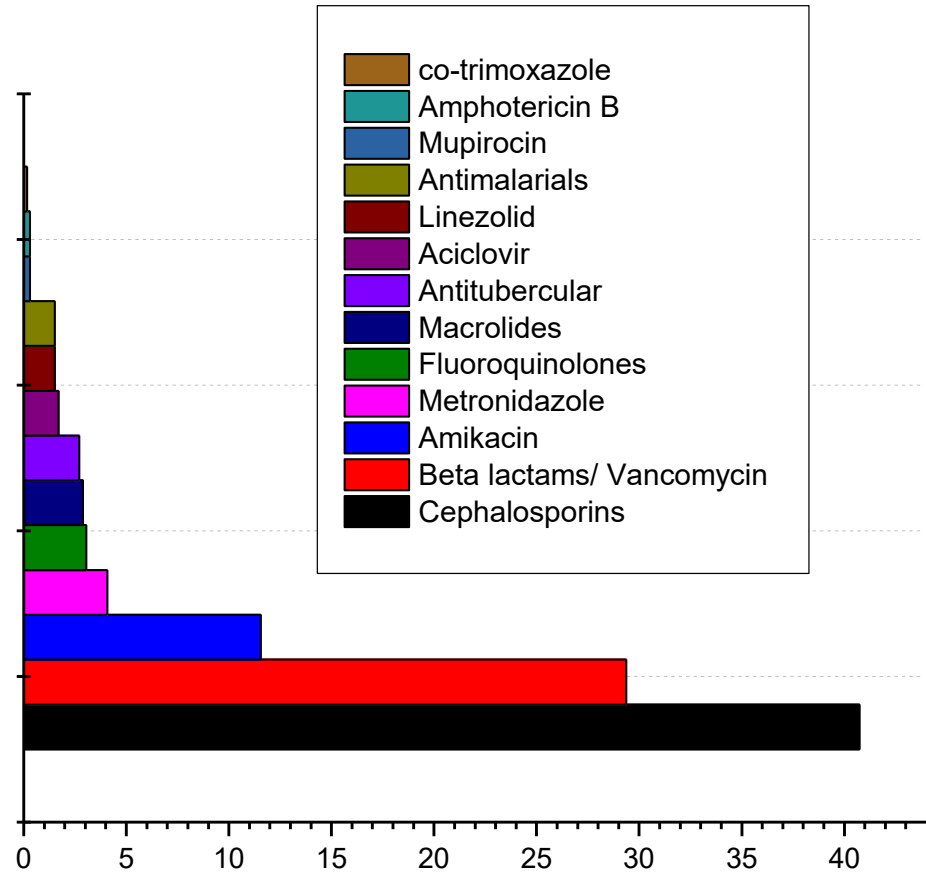

Figure 1. Percentage frequency of use of individual groups of antibiotics in 292 children $(n=589)$

The highest number of antibiotic prescriptions were from the cephalosporin class $(240 ; 40.74 \%)$ while cotrimoxazole, amphotericin B and mupirocin $(0.84 \%$ each) were used sparingly. Ceftriaxone was the single most frequently prescribed drug (33.61\%). Among the 240 instances of cephalosporin use, the highest prescribed was ceftriaxone $(198 ; 82.5 \%)$ whereas cefalexin $(0.16 \%)$ and ceftazidime $(0.16 \%)$ were used the least. The majority of the cephalosporins were prescribed for respiratory tract infections $(62 ; 25.83 \%)$ and the least for soft tissue infections ( $3 ; 1.25 \%)$. Penicillins, other $\beta$ lactams, and vancomycin accounted for 173 (29.37\%) instances. Co-amoxiclav (12.56\%) and ampicillin (4.75\%) were prescribed more frequently; whereas benzathine penicillin and imipenem $(0.16 \%$ each) were used sparingly. Highest numbers in this group were prescribed for respiratory tract infections (34.10\%) and the lowest for skin and soft tissue infections (1.73\%). Fig. 1 and Table 3 provide a system-wise break-up of the 589 instances of antibiotic use.

Overall, the highest numbers of antibiotic agents were prescribed for respiratory tract infections (154, 26.15\%) followed by infections involving the central nervous system (CNS) (106, 17.99\%) and the genitourinary tract $(89,15.11 \%)$. Among the total number of antibiotic prescriptions for respiratory tract infections; the highest numbers were prescribed from other $\beta$-lactam group followed by cephalosporins and aminoglycosides. For CNS infections, highest numbers were prescribed from cephalosporin class followed by other $\beta$ lactams and antitubercular drugs. For fever without specification of cause, antibiotics prescribed (6.28\%) included cephalosporins, other $\beta$-lactams, and amikacin.
It was found 109 (37.32\%) that prescriptions contained a single antibiotic agent, 121 (41.43\%) contained two and $62(21.23 \%)$ contained either three or more than three antibiotic agents.

Analyzing the dosage forms used, it was observed that the majority $(478 ; 81.15 \%)$ of the antibiotics were administered intravenously and the rest by oral route (111; 18.84\%). Only mupirocin was used topically. The great majority $(583 ; 98.98 \%)$ were prescribed in generic name. The several brand name prescriptions included ATD combinations, piperacillin-tazobactam, and ceftriaxone in a single instance.

In most children (84.89\%) antibiotic therapy was empirical and based on clinical judgment. In $15.11 \%$ cases, treatment was guided by results of laboratory tests, including $28(9.58 \%)$ instances of blood culture and 12 (4.1\%) of cerebrospinal fluid examination. Peripheral blood smears were examined in suspected malaria cases. Only 9 specimens out of 28 sent for blood culture showed positive results, with Staphylococcus aureus isolated in five.

ADRs, of at least "possible" causality, were noted in 5 $(1.71 \%)$ children, of which half were cutaneous reactions and the rest loose stools. Vancomycin was responsible for skin rash in 4 cases, while ampicillin was responsible for loose stool in 3 cases and amoxicillin in 1 case. Although the reactions were not deemed to be serious, vancomycin was replaced by suitable alternatives in the skin rash cases. Out of 292 patients studied most were either discharged $(234 ; 80.13 \%)$ or shifted to the PICU $(53 ; 18.15 \%)$. However, 5 babies $(1.71 \%)$ in the study cohort died from their illness. ADRs were not 
implicated in any of the instances of death or worsening of disease.

\section{DISCUSSION}

In this investigation, around $63 \%$ of the subjects were male. These statistic figures are extensively like reports from open public hospitals in other developing countries, for example, Ethiopia [15-17] and Nepal [18]. The most widely indication for antibiotic use in our series was pneumonia (10.2\%), trailed by other lower respiratory tract diseases (9.4\%). Among the antibiotics prescribed for respiratory tract diseases; the majority were cephalosporins or different $\beta$ lactams followed by vancomycin and amikacin. An investigation did in Kathmandu Valley, Nepal18, also observed that pneumonia was the most common diagnosis for in hospital antibiotic use.

In this study, cephalosporins (40.74\%) were most generally prescribed antibiotics followed by other members of the $\beta$-lactam class and aminoglycosides. Ceftriaxone (33.61\%), Co-amoxiclav (12.56\%) and amikacin (11.54\%) were most frequently prescribed.

The Kathmandu Valley study[18] announced that cephalosporins were the most commonly prescribed antibiotics followed by penicillins. Among cephalosporins, ceftriaxone was frequently prescribed. An investigation did in a tertiary hospital in Bangladesh report that ampicillin, gentamicin, amoxicillin, cloxacillin and ceftriaxone were prescribed frequently[19]. A comparative report did in eastern nepal reported that gentamicin, ampicillin, crystalline penicillin, and cefotaxime were the most commonly prescribed antibiotics [20]. A study in Chandigarh by sharma et al and deshmukh et $a /[21,22]$ demonstrated that aminoglycosides (amikacin), cephalosporins (cefotaxime), quinolones (ciprofloxacin), and cloxacillin were most much of the time prescribed. Thus, so far as the pattern of antibiotic use is concerned, our experience broadly conforms to that of other authors reporting prescribing trends for inpatients in similar socio-economic settings. It is noteworthy that our hospital does not have a standing infection control committee or antibiotic use policy that would have constrained choice of drugs.

Percentage of encounters with an antibiotic prescribed (almos $80 \%$ in our case) and normal number of antibiotics per prescription have been considered as essential markers for rational use of medicines in out-patient primary care23. To minimize the risk of drug-drug interactions, development of bacterial resistance and costs, it is preferable to keep the number of drugs per prescription as low as possible. In our study, around $60 \%$ prescriptions contained either two or more than two antibiotics, with the median number at two. Prajapati and Bhatt [7], announced that mean number of drugs per prescription was 1.97 and number of prescriptions with two or more antibiotic agents was around $71 \%$. sharma et al[21]. Observed that prescribing of two anti- biotics was common (around 60\%) in pediatric cases. However, the study carried out in Kathmandu Valley18 reported that the significant portion (93\%) of patients was prescribed one antibiotic. Thus, prima facie the situation in India, and particularly in our setting, offers scope for improvement.

We found that $81.15 \%$ antibiotic agents were prescribed by parenteral routes, while only $18.84 \%$ were for oral utilize; $98.98 \%$ were prescribed in generic name. The prevalence of parenteral administration can be explained in the context of inpatients that are likely to be more seriously ill than patients who can be managed on ambulatory basis. Still the figure for injections appears to be high and calls for investigation through prescription audit and formulation of antibiotic guidelines. Comparative concerns have been voiced in a recent latvian investigation on antibiotic usage among hospitalized children, including neonates[24]. The near $100 \%$ generic prescribing is a desirable situation. We feel that this owes to a large extent on local government policy that seeks to enforce generic prescribing in public hospitals.

Ideally, in any large hospital, antibiotic prescribing should be governed by appropriate guidelines supported by antibiotic resistance observation. Unfortunately, our institution currently does not have an active surveillance program or updated guidelines.In the considerable dominant part of cases, initial drug choice was empirical and antibiotic treatment was guided by the results of laboratory tests in just $15.11 \%$ cases; which are far from satisfactory. Furthermore, the low yield of culture tests, as noted in our case, often frustrate clinicians. A similar circumstance is by all accounts winning in the settings for prior examinations[18]. This is a grossly undesirable situation since antibiotic use guidelines supported through appropriate surveillance is regarded as a fundamental prerequisite for rational antibiotic use[25]. Notwithstanding continuous spread of antibiotic resistance coupled with declining rate of new antibiotic improvement a lot of emphasis is being accorded of late to antibiotic stewardship programs (ASPs) in hospitals[26]. However, although ASPs can be effective in enhancing the quality and safety of antibiotic prescribing for hospitalized patients, there are unique issues relevant to children and pediatric ASPs are still in their infancy $[27,28]$.

In our study, suspected ADRs were noted in just $1.71 \%$ cases and were prevalently cutaneous reactions or loose stool. Thus, the ADR burden was quite manageable. Priyadharsini et al [12] in their study on ADRs in pediatric patients reported that antibiotics were responsible for a large share of the burden and urticaria and different rashes were the most common ADRs attributable to antibiotics. The hospital being part of an ADR Monitoring Center under PvPI, these reports were properly submitted to PvPI. However, as yet there have no feedback sessions in this regard for the physicians 


\section{concerned.}

The present examination has its share of limitations. For logistical reasons, subject recruitment was not done consistently basis and sampling was purposive rather than random. In any case, the prospective data collection has ensured that for subjects recruited we have reliable data. Neonates and PICU patients were not a part of the study, but drug use patterns in such children also need to be addressed.

\section{CONCLUSION}

Nevertheless, in conclusion, we can state that this examination profiles antibiotic use in hospitalized children in our organization and the situation is likely to be representative of other public hospitals catering to poor patients. Utilization of well?known antibiotics and generic prescribing are satisfactory trends noted. On the flip side, numerous antibiotics in the similar patient and commonplace utilization of injections are matters of concern. Furthermore, decision of anti-microbial being generally empirical there may be a tendency to use more expensive cephalosporins in lieu of less expensive drugs.

Suggestions: We hope that these findings can be utilized in conjunction with antibiotic resistance surveillance data in formulating antibiotic use guidelines for pediatric wards. Medication utilize overviews in different settings, for example, pediatric OPD and PICU are required before wandering into the improvement of comprehensive pediatric anti-microbial utilize rules.

\section{REFERENCES}

1) The world medicines situation 2011. 3rd ed. Geneva: WHO; 2011.

2) World Health Day. Director General Statement. Antimicrobial resistance: no action today, no cure tomorrow; 2011. Available from: http:// www.who.int/world-health-day/2011.

3) Chandy SJ, Thomas K, Mathai E, Antonisamy B, Holloway KA, Stalsby Lundborg C. Patterns of antibiotic use in the community and challenges of antibiotic surveillance in a lower-middle-income country setting: a repeated cross-sectional study in Vellore, South India. Journal of Antimicrobial Chemotherapy. $2012 ; 68(1): 229-36$.

4) Wattal C, Goel N. Tackling antibiotic resistance in India. Expert review of anti-infective therapy. 2014;12(12):1427-40.

5) Chatterjee D, Sen S, Begum SA, Adhikari A, Hazra A, Das AK. A questionnaire-based survey to ascertain the views of clinicians regarding rational use of antibiotics in teaching hospitals of Kolkata. Indian journal of pharmacology. 2015;47(1):105.

6) Kanungo S, Mahapatra T, Bhaduri B, Mahapatra S,
Chakraborty ND, Manna B, Sur D. Diarrhoea-related knowledge and practice of physicians in urban slums of Kolkata, India. Epidemiology \& Infection. 2014;142(2):314-26.

7) Prajapati V, Bhatt JD. Study of prescribing pattern of antimicrobial agents in the pediatric wards at tertiary teaching care hospital, Gujarat. Indian J Pharm Sci Res. 2012;3:2348-55.

8) McGowan Jr JE. Antimicrobial resistance in hospital organisms and its relation to antibiotic use. Reviews of infectious diseases. 1983;5(6):1033-48.

9) Reichler MR, Allphin AA, Breiman RF, Schreiber JR, Arnold JE, McDougal LK, et al. The spread of multiply resistant Streptococcus pneumoniae at a day care center in Ohio. Journal of Infectious Diseases. 1992;166(6):1346-53..

10)Aagaard L, Hansen EH. Adverse drug reactions reported for systemic antibacterials in Danish children over a decade. British journal of clinical pharmacology. 2010;70(5):765-8.

11) Impicciatore $P$, Choonara I, Clarkson A, Provasi D, Pandolfini C, Bonati M. Incidence of adverse drug reactions in paediatric in/out-patients: a systematic review and meta-analysis of prospective studies. British journal of clinical pharmacology. 2001;52 (1):77-83.

12) Priyadharsini R, Surendiran A, Adithan C, Sreenivasan S, Sahoo FK. A study of adverse drug reactions in pediatric patients. Journal of pharmacology \& pharmacotherapeutics. 2011;2(4):277.

13)Baidya S, Hazra A, Datta S, Das AK. A study of antimicrobial use in children admitted to pediatric medicine ward of a tertiary care hospital. Indian journal of pharmacology. 2017;49(1):10.

14) Pharmacovigilance Programme of India. Suspected Adverse drug Reaction Reporting Form. Available from: $\quad$ http://www.ipc.gov.in/PvPI/ ADRReportingForm.pdf. [Last accessed on $2013 \mathrm{Apr}$ 20].

15)Zaki S. Adverse drug reaction and causality assessment scales. Lung India. 2011;28(2):152.

16) Osowicki J, Gwee A, Noronha J, Palasanthiran P, McMullan B, Britton PN, Isaacs D, Lai T, Nourse C, Avent $M$, Moriarty P. Australia-wide point prevalence survey of the use and appropriateness of antimicrobial prescribing for children in hospital. The Medical Journal of Australia. 2014;201(11):657-62.

17) Woldu MA, Suleman S, Workneh N, Berhane H. Retrospective study of the pattern of antibiotic use in Hawassa University referral hospital pediatric ward, Southern Ethiopia. Journal of Applied Pharmaceutical Science. 2013;3(2):93. 
18) Palikhe N. Prescribing pattern of antibiotics in pediatric hospital of Kathmandu valley. Age (mean \pm SD years). 2004;4:3-9.

19)Akter FU, Heller D, Smith A, Rahman MM, Milly AF. Antimicrobial use in paediatric wards of teaching hospitals in Bangladesh. Mymensingh medical journal: MMJ. 2004;13(1):63-6..

20) Rauniar GP, Naga Rani MA, Das BP. Prescribing pattern of antimicrobial agents in paediatric inpatients. J Nepalgunj Med. Coll. 2000;3:34-7.

21) Sharma D, Reeta KH, Badyal DK, Garg SK, Bhargava VK. Antimicrobial prescribing pattern in an Indian tertiary hospital. Indian journal of physiology and pharmacology. 1998;42(4):533-7.

22) Deshmukh VS, Khadke VV, Patil AW, Lohar PS. Study of prescribing pattern of antimicrobial agents in indoor patients of a tertiary care hospital. International Journal of Basic \& Clinical Pharmacology. 2017;2(3):281-5..

23)World Health Organization Action Programme on Essential Drugs. How to Investigate drug Use in Health Facilities: Selected drug Use Indicators. Geneva: WHO; 1993

24)Sviestina I, Mozgis D. Antimicrobial usage among hospitalized children in Latvia: A neonatal and pediatric antimicrobial point prevalence survey. Medicina. 2014;50(3):175-81.

25) Versporten A, Sharland M, Bielicki J, Drapier N, Vankerckhoven V, Goossens H. The antibiotic resistance and prescribing in European Children project: a neonatal and pediatric antimicrobial webbased point prevalence survey in 73 hospitals worldwide. The Pediatric infectious disease journal. 2013;32(6):e242-53.

26) Walia K, Ohri VC, Mathai D. Antimicrobial stewardship programme (AMSP) practices in India. The Indian Journal of Medical Research. 2015;142(2):130.

27) Newland JG, Banerjee R, Gerber JS, Hersh AL, Steinke L, Weissman SJ. Antimicrobial stewardship in pediatric care: strategies and future directions. Pharmacotherapy: The Journal of Human Pharmacology and Drug Therapy. 2012 Aug 1;32(8):735-43.

28)Johnson $C L$, Jain $M$, Saiman $L$, Neu N. Antimicrobial stewardship in pediatric post-acute care facilities. American journal of infection control. $2018 ; 46$ (4):468-70.

How to Cite this article: Praveena Gungam, Y. Sunil Kumar Yadav, Sunil Junapudi. Antibiotic use in pediatric infections: A study in tertiary care hospital. Int. j. clin. biomed. res. 2018;4(4): 26-32. 